\title{
Estimation of Mutagenic/Carcinogenic Potential of Environmental Contaminants by Ion-Molecule Reactions and Tandem Mass Spectrometry
}

\author{
Jody A. Freeman, Jodie V. Johnson, Mark E. Hail*, and Richard A. Yost \\ Department of Chemistry, University of Florida, Gainesville, Florida, USA
}

Douglas W. Kuehl

U.S. Environmental Protection Agency, Duluth, Minnesota, USA

The ability to produce and detect products of model DNA/carcinogen ion-molecule reactions is demonstrated in the ion source and the collision cell of a triple quadrupole tandem mass spectrometer. Reaction between adenine and benzoyl chloride in the ion source is shown to produce the DNA adduct benzoyl adenine. The daughter ion mass spectrum of the reaction product is compared to that of the synthesized standard. Mass chromatograms of the reaction between mass-selected pyridine ions and various analytes eluting from a GC column into the collision cell are demonstrated and illustrate the ability to detect only the GC eluates that react with pyridine. This technique could provide a rapid and sensitive method for screening complex environmental samples for carcinogens, as well as for estimating the relative mutagenic/carcinogenic potential of environmental contaminants. ( $\mathrm{J}$ Am Soc Mass Spectrom 1990, 1, 110-115)

$\mathrm{P}$ otentially mutagenic and carcinogenic chemical contaminants are recognized to be reactive electrophiles capable of modifying biological macromolecules, such as DNA [1]. These compounds, however, represent only a small portion of the total number of contaminants in the typical environmental sample. Accurate evaluation of these chemicals to provide data for human and ecosystem risk assessment depends upon analytical procedures to isolate, identify, and quantify each chemical and to provide a comparative measure of electrophilic reactivity. Conventional biological methods for estimating mutagenic potential, such as the Ames bacteriological assay and animal assays, tend to be time-consuming (e.g., 2 days for the Ames test) [2] and operate in a batch mode (i.e., not online with chromatographic separation). Hence these methods are limited to single chemical evaluations or, in the case of environmental extracts, evaluation of mixtures of generally unknown composition.

Alternatively, chemical methods have been developed to estimate mutagenicity by determining the potential for covalent bonding of electrophiles to DNA or model DNA molecules, such as the nucleophiles 4(4-nitrobenzyl)pyridine [3], 4-nitrophenol [4, 5], or 3,4dichlorobenzenethiol [6]. Although these methods are less time consuming (1-2 h), they are still limited to

\footnotetext{
* Current address: Finnigan MAT, 355 River Oaks Parkway, San Jose, CA 95134-1991.

Address reprint requests to Richard A. Yost, Department of Chemistry,
} University of Florida, Gainesville, FL 32611, USA. batch mode. Also, offline synthesis of DNA adducts by electrochemical oxidation and subsequent analysis by mass spectrometry has been reported [7]. Furthermore, the gas-phase reaction of pyridine and guanine with polynuclear aromatic hydrocarbons (PAHs) in the ion source of a tandem mass spectrometer is under investigation elsewhere [8]. In addition, gas-phase synthesis of alkylated DNA nucleosides has been studied in the ion source of a mass spectrometer [9]. These latter two methods can be interfaced with chromatographic separation techniques.

The most useful tool for the efficient and rapid characterization of chemical contaminants in environmental samples remains the computerized gas chromatography/mass spectrometry (GC/MS) system. Because of this, a third approach to the evaluation of potential carcinogens and mutagens has been to use computer predictions of chemical reactivity from quantitative structure-activity relationship (QSAR) models $[10,11]$. The U.S. Environmental Protection Agency is using QSAR-GC/MS in the premarket notification process for the registration of chemicals and for the assessment of contaminants at hazardous waste sites. Disadvantages of QSAR-GC/MS are that, first, each contaminant must be identified correctly and, second, the tremendous volumes of data that can be rapidly produced by a GC/MS system can quickly overwhelm data reviewers.

There is a substantial need for a rapid, sensitive, cost-effective technique, not only to screen complex 
environmental samples for contaminants but also to estimate the relative mutagenic/carcinogenic potential of chemicals. Here we report our preliminary evaluation of ion-molecule reactions to estimate the mutagenic/carcinogenic potential of environmental contaminants. Ion-molecule reactions are performed in the ion source or collision cell of a tandem quadrupole mass spectrometer, with DNA bases or model nucleophiles used to represent biological macromolecules. The electrophilic mixtures are introduced into the ion source or collision cell via GC. Model DNA-carcinogen reactions are performed in a tandem mass spectrometer, which can minimize the number of mass spectrometric peaks and identify possible carcinogens. Furthermore, the potential is demonstrated to correlate the observed reactivity of electrophiles and model bases with the relative mutagenicities determined by the Ames test.

\section{Experimental}

\section{Reactions in the Ion Source}

The reaction of adenine with benzoyl chloride was studied in the ion source of a Finnigan MAT (San Jose, CA) TSQ 45 triple quadrupole mass spectrometer. Adenine was introduced continuously into the ion source from a solids probe heated at a constant temperature of $140^{\circ} \mathrm{C}$. Benzoyl chloride was introduced via a Finnigan MAT 9610 gas chromatograph into the ion source by a $\mathrm{J} \& \mathrm{~W}$ Scientific (Folsom, CA) 3m DB-1 (0.2 mm i.d., $0.25 \mu \mathrm{m}$ film thickness) capillary column heated isothermally at $50{ }^{\circ} \mathrm{C}$. The injection port, transfer line, and interface were all held at $250{ }^{\circ} \mathrm{C}$. All species in the ion source were ionized by methane chemical ionization (CI) at a pressure of 0.40 torr indicated with a Granville Phillips (Boulder, $\mathrm{CO}$ ) thermogauge. Mass spectra were obtained at an electron energy of $100 \mathrm{eV}$, an emission current of $300 \mu \mathrm{A}$, and ion source temperature of 190 ${ }^{\circ} \mathrm{C}$. Positive ion $\mathrm{CI}(\mathrm{PCI})$ and electron capture negative ion $\mathrm{CI}(\mathrm{ECNCI})$ spectra were acquired with \pm 3 $\mathrm{kV}$ dynodes for pulsed positive ion-negative ion $\mathrm{CI}$ (PPINICD), and electron multiplier voltages of -800 and $-1500 \mathrm{~V}$ for mass spectrometry and tandem mass spectrometry (MS/MS), respectively. Daughter ion spectra were acyuired at a collision energy of 28 $\mathrm{eV}$ and an indicated collision cell pressure of 1.0 mtorr argon. The collision cell pressure was measured with a Teledyne-Hastings-Raydist (Hampton, VA) DV8 thermocouple gauge. One-microliter splitless injections of $500 \mathrm{ng} / \mu \mathrm{L}$ benzoyl chloride in methanol were made. Adenine and $N^{6}$-benzoyl adenine were obtained from Sigma Chemical Company (St. Louis, MO) and benzoyl chloride from Eastman Kodak Company (Rochester, NY).

\section{Reactions in the Collision Cell}

The reaction of pyridine with various electrophiles was studied in the collision cell of a Finnigan MAT TSQ 70 triple quadrupole mass spectrometer. The concepts, instrumentation, and performance of GC introduction into the second quadrupole (collision cell, Q2) of a tandem mass spectrometer were described previously [12]. Pyridine was introduced continuously into the ion source by a Granville Phillips fine-metering valve, and the analytes were introduced into the collision cell by means of a J\&W Scientific $6 \mathrm{~m}$ DB- $5(0.178 \mathrm{~mm}$ i.d., $0.4 \mu \mathrm{m}$ film thickness) capillary column. The $\mathrm{M}^{+}$or $(\mathrm{M}+\mathrm{H})^{+}$ion of pyridine, produced under methane $\mathrm{CI}$ conditions, was mass-selected by $Q 1$ and reacted with the neutral analytes in the collision cell at low collision energies of approximately $1 \mathrm{eV}$. Product ions were then mass-analyzed in Q3. The Varian 3400 gas chromatograph was operated with a split ratio of $30: 1$, an injection port temperature of $250^{\circ} \mathrm{C}$, and a transfer line temperature of $170^{\circ} \mathrm{C}$. The $\mathrm{GC}$ oven was temperatureprogrammed from $25^{\circ} \mathrm{C}$ to $160^{\circ} \mathrm{C}$ at $40^{\circ} \mathrm{C} / \mathrm{min}$ after an initial hold period of $1.2 \mathrm{~min}$.

Pyridine was ionized under PCI conditions on the TSQ 70, at an ion source pressure of 1.6 torr $\mathrm{CH}_{4}$ indicated with a Granville Phillips convectron gauge, an electron energy of $100 \mathrm{eV}$, an ion source temperature of $150^{\circ} \mathrm{C}$, a manifold temperature of $75^{\circ} \mathrm{C}$, and an emission current of $200 \mu \mathrm{A}$. Product ion spectra were acquired with dynode voltages of $\pm 5 \mathrm{kV}$ and an electron multiplier voltage of $-1100 \mathrm{~V}$. The collision energy, Q2 If potential, lenses immediately before $Q 2$, and all lenses following $Q 2$ were optimized for collision cell reactions. This was accomplished by continuously introducing allyl chloride into the collision cell via the collision gas inlet and a Negretti (Hampshire, England) fine-metering needle valve and then optimizing the lenses for maximum transmission of the reactant and product ions. The collision energy and Q2 $\mathrm{rf}$ potential were adjusted for maximum product ion formation.

Test mixtures were prepared in heptane $(6 \mu \mathrm{g} / \mu \mathrm{L})$ containing six components (allyl chloride, allyl bromide, allyl isothiocyanate, ethylbenzene, styrene, and hexachlorobutadiene) or two components (propyl chloride and propyl bromide). One-microliter injections (30:1 split) of the mixtures were made onto the GC column. Allyl bromide, allyl chloride, propyl bromide, and propyl chloride were purchased from Eastman Kodak, hexachlorobutadiene and allyl isothiocyanate from Aldrich Chemical Company (Milwaukee, WI), and ethylbenzene, styrene, and pyridine from Fisher Scientific (Fair Lawn, NJ).

\section{Results and Discussion}

\section{Reactions in the Ion Source}

The positive and negative ion $\mathrm{CI}$ mass spectra (Figure 1) obtained from the reaction of adenine and benzoyl chloride in the ion source demonstrate the ability to produce DNA adducts in the ion source. In all the spectra shown, the nucleophile is labeled $\mathrm{N}$, the electrophile $\mathrm{E}$, and the DNA reaction product $\mathrm{P}$. The $\mathrm{m} / \mathrm{z}$ 

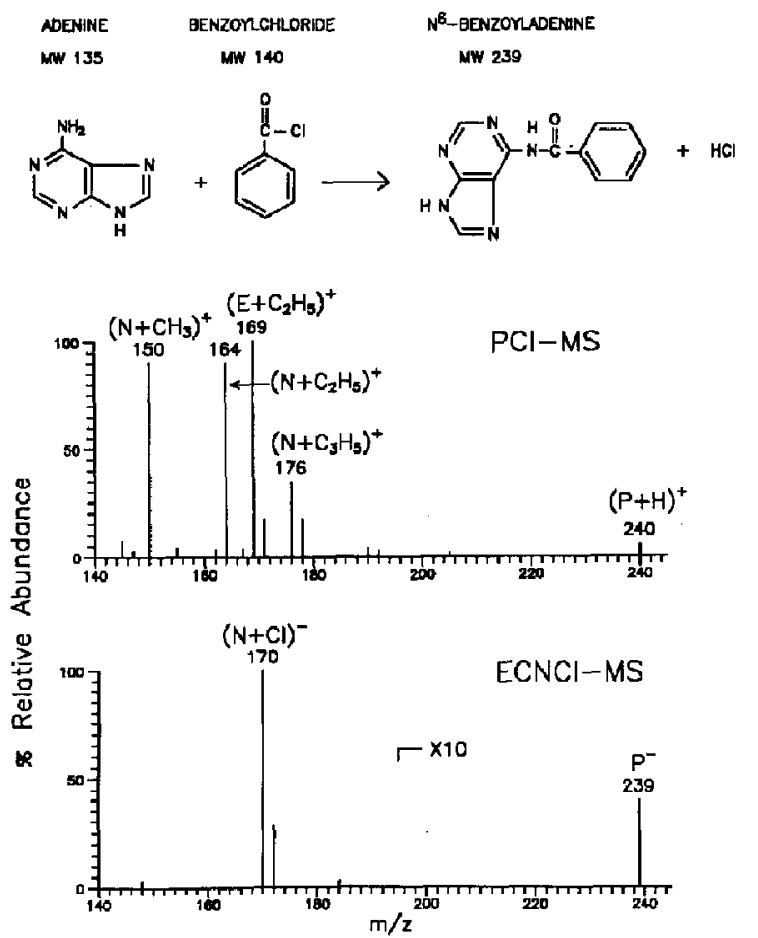

Figure 1. PCI and ECNCI mass spectra of the adenine-benzoyl chloride reactions in the ion source of the TSQ 45.

240 peak in the $\mathrm{PCI}$ mass spectrum is the $(\mathrm{P}+\mathrm{H})^{+}$ of the reaction product, and $m / z 239$ in the ECNCI mass spectrum is the $\mathrm{P}^{-}$of the product. In addition, methane adduct ions $(\mathrm{m} / \mathrm{z} 150,164,169,176)$ of both reactants are observed in the positive mode, and chloride adduct ions $(\mathrm{m} / \mathrm{z} 170,172)$ of the nucleophile are observed in the negative mode. The daughter ion spectra (shown in Table 1 ) of the $(\mathbf{P}+\mathbf{H})^{+}$and $\mathrm{P}^{-}$adduct ions produced in the ion source provide further information for identification of the reaction product. It is interesting to observe that the positive and negative daughter ions complement one another; $m / z 240$ positive ion fragments to the benzoyl ion, whereas $m / z 239$ negative ion fragments to the adenine ion. Further confirmation is possible by comparison of the daughter ion spectra obtained from the adduct ion and the synthesized standard (Table 1). The differences observed, especially in the ratios of the daughter ion abundances to that of the parent ion remaining after collision-activated dissociation (CAD) may arise from differences in internal energy of the parent ions. Further studies are needed to address this issue.

\section{Reactions in the Collision Cell}

The PCI mass spectrum of the allyl chloride-pyridine ion reaction in the collision cell is compared to one obtained from the ion source (Figure 2). In the ion source mass spectrum, the $m / z 120$ positive ion peak can correspond to the $\left(\mathrm{N}+\mathrm{C}_{3} \mathrm{H}_{5}\right)^{+}$methane $\mathrm{Cl}$ adduct ion of
Table 1. Comparison of daughter ion spectra for the product ions resulting from ion source reactions between adenine and benzoyl chloride and the corresponding synthesized standard ions of $N^{6}$-benzoyl adenine

\begin{tabular}{|c|c|c|c|}
\hline$m / z$ & Structure & $\begin{array}{l}\% \text { RA of ion- } \\
\text { molecule reaction }\end{array}$ & $\begin{array}{c}\% \text { RA of synthesized } \\
\text { standard }\end{array}$ \\
\hline
\end{tabular}

Daughter ion spectra of $\mathrm{m} / 2240^{+}$

$\begin{array}{rcrr}240 & {[\mathrm{P}+\mathrm{H}]^{+}} & 27 & 54 \\ 105 & \mathrm{C}_{6} \mathrm{H}_{5} \mathrm{CO}^{+} & 100 & 100 \\ 77 & \mathrm{C}_{6} \mathrm{H}_{5}^{+} & 9 & 8\end{array}$

Daughter ion spectra of $m / z 239$

$\begin{array}{rcrr}239 & \mathrm{P}^{-} & 6 & 72 \\ 134 & {[\mathrm{~N}-\mathrm{H}]^{-}} & 100 & 100 \\ 133 & {[\mathrm{~N}-2 \mathrm{H}]^{-}} & - & 32\end{array}$

all daughter ions $>4 \%$ are reported.

RA $=$ relative abund ance.

pyridine and/or the reaction product of pyridine $\mathrm{M}^{+}$ ion and allyl chloride. The collision cell mass spectrum, however, is simpler and less ambiguous because only one ion (pyridine $\mathrm{M}^{+}$) is present in the collision cell to react with the neutral analytes eluting from the gas chromatograph. Investigation of $\mathrm{Q} 2$ reactions with the $\mathrm{M}^{+}$and $(\mathrm{M}+\mathrm{H})^{+}$ions of pyridine indicated that only the $\mathrm{M}^{+}$ion reacted with the allyl chloride; thus, only reactions with the $\mathrm{M}^{+}$ion of pyridine were further evaluated.
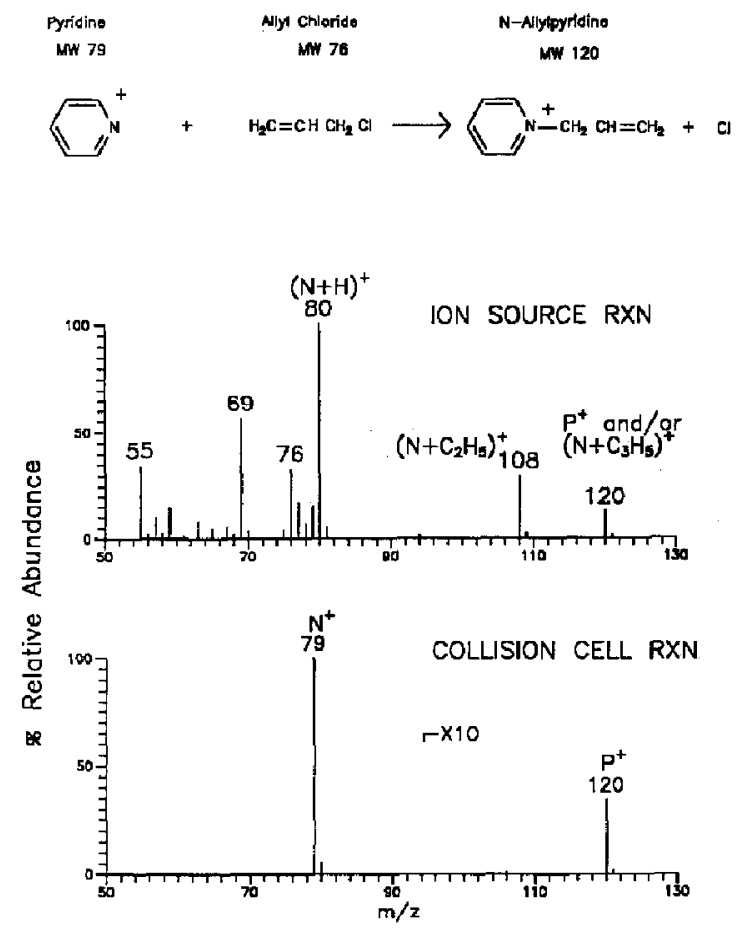

Figure 2. Comparison of the ion source and collision cell PCI mass spectra of the pyridine-allyl chloride reaction. 


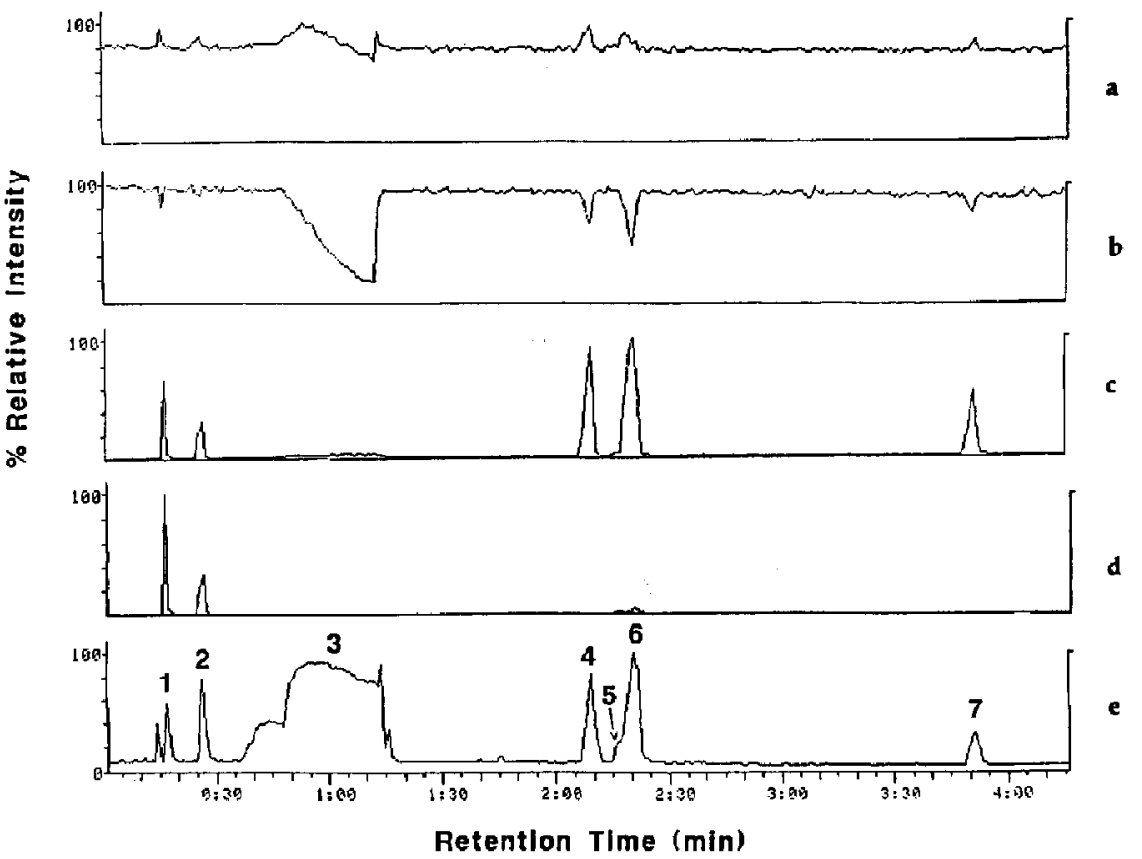

Figure 3. Mass chromatograms (a), (b), (c), and (d) are the result of $Q 1$ mass-selecting the $m / z 79$ ion of pyridine to react with various analytes from the gas chromatograph: (a) RIC, (b) trace of $m / z 79$ ion, (c) the RIC minus $m / z$ 78-80 ions, (d) the trace of only the $m / z 120$ ion. Mass chromatogram (e) is a result of $Q 1$ mass-selecting the $m / z 17$ ion of methane to react with the GC eluents. The GC sample is a mixture of (1) allyl chloride, (2) allyl bromide, (3) heptane [solvent], (4) ethylbenzene, (5) allyl isothiocyanate, (6) styrene, and (7) hexachlorobutadiene.

Figure 3 illustrates mass chromatograms obtained by alternately mass-selecting the $m / z 79$ ion of pyridine $\left(\mathrm{M}^{+}\right)$and $m / 217$ ion of methane $\left(\mathrm{CH}_{5}^{+}\right)$with Q1 to react with the components of the test mixture eluting from the gas chromatograph into the collision cell. Thus, mass chromatograms can be obtained that either depict only reactive compounds (reaction with pyridine $\mathrm{M}^{+}$) or depict all the compounds in the mixture (protonated by $\mathrm{CH}_{5}^{+}$). The $\mathrm{CI}$ mass spectra that result from reaction with $\mathrm{CH}_{5}^{+}$can help in the identification of the reactive compounds. Chromatograms (a), (b). (c), and (d) of Figure 3 result from mass-selecting miz 79 ion to react with the analyte. Chromatogram (a) displays the reconstructed ion current (RIC) and is relatively uninformative. Chromatogram (b) displays the trace of the $m / z 79$ positive ion and demonstrates the difficulty of monitoring the loss of reactant ion, because loss of $m / z 79$ can also be the result of ion-molecule reactions (e.g., charge exchange) with components eluting from the gas chromatograph that do not result in the desired product ions. Chromatogram (c) is the RIC minus signals due to the $\mathrm{m} / \mathrm{z}$ 78-80 ions; it illustrates the capability to monitor eluents that react in any way with the reactant ion. Chromatogram (d) is the trace of $\mathrm{m} / \mathrm{z} \mathbf{1 2 0}$ ion; this permits one to screen for GC eluents that react with pyridine to produce a specific product ion characteristic of a class of environmental contaminants, in this case, the $\mathrm{m} / \mathrm{z}$. 120 product ion, which is characteristic of allyl halides.
Chromatogram (e) displays the RIC resulting from the mass-selection of the $m / z 17$ ion of methane $\left(\mathrm{CH}_{5}^{+}\right)$and subsequent reaction with the eluting components. This chromatogram illustrates the ability to detect all compounds in the GC sample regardless of whether the compounds react with the $m / z 79$ ion and may be used to determine the concentration of each eluent.

Figure 3 contains several peaks that need further explanation. The small peak in chromatogram (e) before allyl chloride (peak 1) has been identified as that of pentane (an impurity in the heptane) from the pentane mass spectrum and GC retention time. It was not expected that heptane would react with pyridine; the large decrease in the $m / z 79$ ion (b) at the retention time of heptane results when the pyridine ion abstracts a hydrogen from heptane to form an ion of $m / z$ 80, protonated pyridine. For this reason, the $m / z$ 80 ion was excluded in chromatogram (c). Although ethylbenzene (4) and styrene (6) are noncarcinogens without metabolic activation, mass chromatogram (c) includes intense peaks for these two compounds. The mass spectrum of ethylbenzene (MW 106) shows no ion corresponding to a reaction with pyridine, but rather a very abundant ion of $m i z 106$ produced by charge exchange. The mass spectrum of the styrene (MW 104) peak shows ions at $\mathrm{m} / \mathrm{z} 104,183$, and 208, which are the $\mathrm{M}^{+}$ion produced by charge exchange, the pyridine adduct ion, and the styrene dimer ion $\left(\mathrm{M}_{2}^{+}\right)$, respectively. In contrast to the carcinogenic allyl 
Allyl Halides

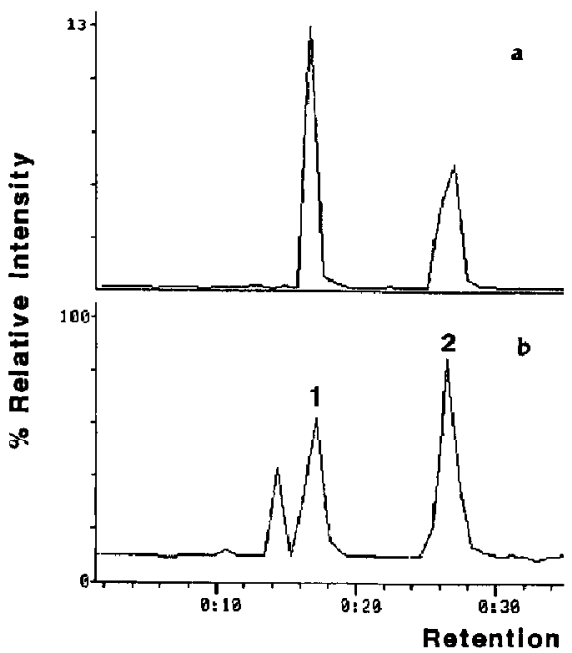

Propyl Halides

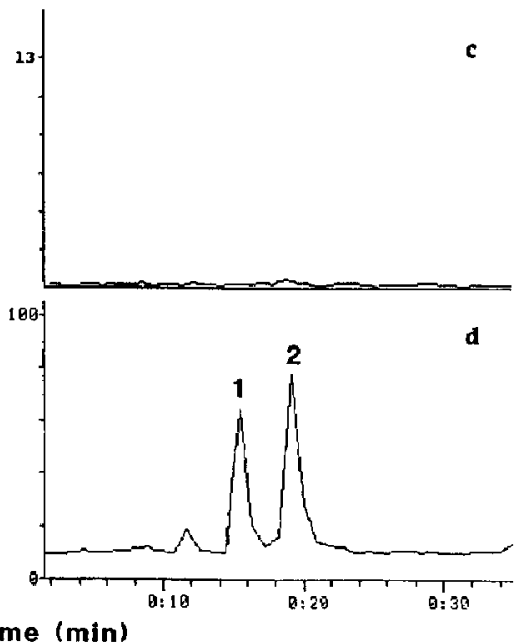

Figure 4. Mass chromatograms comparing Q2 reactions of carcinogenic allyl compounds (a and b) and noncarcinogenic propyl compounds (c and d). Mass chromatograms (a) and (c) are a result of mass-selecting the $m / z 79$ ion of pyridine to react with the GC eluents in the collision cell; (b) and (d) are a result of mass-selecting the $m / z 17$ ion of methane to react with the eluents. The GC eluents are (1) allyl chloride, (2) allyl bromide, (3) propyl chloride, and (4) propyl bromide.

compounds, there is no loss of a leaving group in the styrene-pyridine reaction.

Allyl compounds $\left(\mathrm{C}_{3} \mathrm{H}_{5} \mathrm{X}\right)$ are known carcinogens that react with DNA to form adducts, whereas the analogous propyl compounds $\left(\mathrm{C}_{3} \mathrm{H}_{7} \mathrm{X}\right)$ are noncarcinogenic and therefore should not react with DNA [13]. The results of alternating between the $m / z 79$ ion of pyridine and the miz 17 ion of methane, shown in Figure 4 , are compared for equivalent amounts of the two classes of compounds. The allyl halide chromatograms of Figures $4 a$ and $b$ demonstrate the gas-phase reactivity of the carcinogenic allyl compounds with the positive ion of $m / z 79$. The propyl halide chromatograms of Figures $4 \mathrm{c}$ and $\mathrm{d}$, in contrast, illustrate that no significant reaction with the $m / z 79$ ion is observed for these noncarcinogenic compounds. Note, however, that the reactivity with $\mathrm{CH}_{5}^{+}$is approximately equal for the corresponding allyl and propyl compounds. Thus, monitoring reactions of the $m / z 79$ ion of pyridine relative to the $m / z 17$ ion of methane may aid in estimating the degree of carcinogenicity.

Table 2 compares the collision cell gas-phase reactivity and the Ames test mutagenicity [13] for various carcinogenic and noncarcinogenic compounds. The same order of mutagenicity is predicted; indeed, the correlation of the gas-phase reactivity with the logarithm of the mutagenicity is remarkably good $\left(r^{2}=0.995\right)$. If the mutagenicity of the propyl compounds is assigned a value of 0.1 , then $r^{2}=0.992$. Studies are under way to assess the degree of correlation between gas-phase reactivities and solution-phase estimates of carcinogenicity for a wide range of compounds.

\section{Conclusions}

The ability to produce and detect products of nucleophilic/electrophilic reactions has been demonstrated in the ion source and collision cell of a tandem mass spectrometer. Mass spectra of reactions in the ion source tend to be complicated; furthermore, it is difficult to determine which form of which species is reacting (e.g., $[\mathrm{M}+\mathrm{H}]^{+}, \mathrm{M}^{+}$, fragment ions, or neutral species). Mass spectra of reactions in the collision cell are less complicated, as only a single, mass-selected reactant ion is allowed to interact with the neutral analytes. Tandem mass spectrometric confirmation of the product ion by daughter ion mass spectra is one advantage of reactions in the ion source, because CAD of collision cell reactions cannot be performed without the use of an instrument capable of MS/MS/MS. Investigation into what quantities of analytes are needed to detect products by this technique are under way; the

Table 2. Comparison of collision cell gas-phase reactivity and mutagenicity

\begin{tabular}{lcc}
\hline & Gas-phase reactivity $^{3}$ & Mutagenicity $^{\text {b }}$ \\
\hline Allyl NCS & 1.00 & 1 \\
Allyl Cl & 2.69 & 9 \\
Allyl Br & 5.28 & 700 \\
Propyl Cl & 0.02 & - \\
Propyl Br & 0.14 & - \\
\hline
\end{tabular}

- Values calculated as area of adduct ion per millimole injected and normalized to allyl NCS.

b Values obtained from Ames tests: the propyl analogues possess no alkylating properties or mutagenic activity 113]. 
200-ng quantities reported here provide high signalto-noise ratios for those analytes that react. Previous studies of charge-exchange reactions in the collision cell have yielded full-scan detection limits of $100 \mathrm{pg}$ [12].

The analytical utility of reactions between model DNA bases and electrophiles introduced via a gas chromatograph into the ion source or collision cell of a tandem mass spectrometer could be compared to the online coupling of a gas chromatograph and the Ames test. This technique offers the distinct advantages of being very fast and permits testing of individual components of a complex mixture. These preliminary studies indicate that this method has the potential for wide application in the assessment of carcinogenic and mutagenic potential of environmental contaminants.

\section{References}

1. Miller, J. A. Cancer Res. 1970, 30, 559-576.
2. Ames, B. N. Science 1979, 204, 587-592.

3. Hermens, J.; Busser, F.; Leeuwanch, P.; Musch, A. Toxicol. Environ. Chem. 1985, 9, $219-236$.

4. Agarwal, S. C.; Van Duren, B. L.; Solomon, J. J.; Kline, S. A. Environ. Sci. Technol. 1980, 14, 1249-1253.

5. Chen, A. M.; Carlson, R. E. Anal. Chem, 1981, 53, 1001-1006.

6. Gothe, R.; Calleman, C. J.; Ehrenberg, L.; Wachtmeister, C. A. Ambio 1974, 3, 234-236.

7. Rogan, E. G.; Cavalieri, E. L.; Tibbels, S. R.; Cremonesi, P.; Warner, C. D.; Nagel, D. L.; Tomer, K. B.; Cerny, R. L.; Gross, M. L. J. Am. Chem. Soc. 1988, 110, 4023-4029.

8. Davoli, E.; Cerny, R. L.; Gross, M. L. Ado. Mass Spectrom. 1989, in press.

9. Isern-Flecha, I.; Cooks, R. G.; Chang, C.-J. Manuscript in preparation.

10. Lipnick, R. L. Enoiron. Toxicol. Chem. 1989, 8, 1-12.

11. Turner, L.; Choplin, F.; Dugard, P.; Hermens, J.; Jaeckh, R.; Marsmarun, M.; Roberts, D. Toxicol. In Vitro 1987, 1, 143-171.

12. Hail, M. E.; Berberich, D. W.; Yost, R. A. Anal. Chem. 1989, 61, 1874-1879.

13. Eder, E.; Henschler, D.; Neudecker, T. Xenobiotica 1982, 12, 831-848. 